\title{
IM botulinum toxin-A improves ambulation in severe haemophilia and cerebral palsy
}

\author{
Melanie Bladen, ${ }^{*}$ Karen Edwards, Kate Khair, Eleanor Main
}

Introduction: The use of $\mathrm{BtA}$ in spasticity management is well documented in the literature for the management of hypertonicity in children with cerebral palsy.

Methods: We report a case of a 2-year-old boy with severe haemophilia and cerebral palsy who received intramuscular injections of botulinum toxin-A (BtA) to reduce his spastic diplegia lower limb hypertonicity.

Results: Following treatment, clinical and formal assessments demonstrated positive changes in ambulation, tolerance of orthotics, functional abilities and musculoskeletal range of movement and no adverse bleeding side effects.

Conclusion: The use of BtA should be considered for the management of hypertonicity in children with haemophilia and cerebral palsy.

Key words: haemophilia, cerebral palsy, botulinum toxin-A

Intramuscular botulinum toxin-A (BtA) to produce neuromuscular blockade has been effective in treating spasticity in children with cerebral palsy (CP) [1-3]. The use of $\mathrm{BtA}$ in children with a combination of $\mathrm{CP}$ and

haemophilia has not previously been reported. We report a case of a 2-year-old boy with severe haemophilia and CP who received intramuscular injections of BtA to reduce his spastic diplegia lower limb hypertonicity.

Intramuscular BtA injections are indicated to relieve dynamic spasticity affecting function in the absence of a fixed deformity, or pain, or to facilitate the use of orthotics [4-7]. The literature also supports the use of BtA in children 4 years old or younger in the hope of preventing or delaying deformity $[6,8]$.

\section{Presentation}

\section{Materials and methods}

The 2-year-old boy (for the purpose of this report named Joshua) was examined in February 2008, and assessment included physical examination, video analysis and the Gross Motor Function Measure (GMFM-66). The modified

\footnotetext{
* Melanie Bladen*, Haemophilia Centre Great Ormond St

Hospital for Children NHS Trust. Email: melanie.bladenagosh.nhs.uk
}

Karen Edwards, Movement Disorder Team, Great Ormond Street Hospital for Children NHS Trust, Great Ormond Street London WC1N 3JH

Kate Khair, Nurse Consultant, Haemophilia Centre Great Ormond St Hospital for Children NHS Trust

Eleanor Main, Portex Department, 6th Floor, Cardiac Wing, Institute of Child Health, University College London, 30 Guilford Street, London, WC1N 1EH

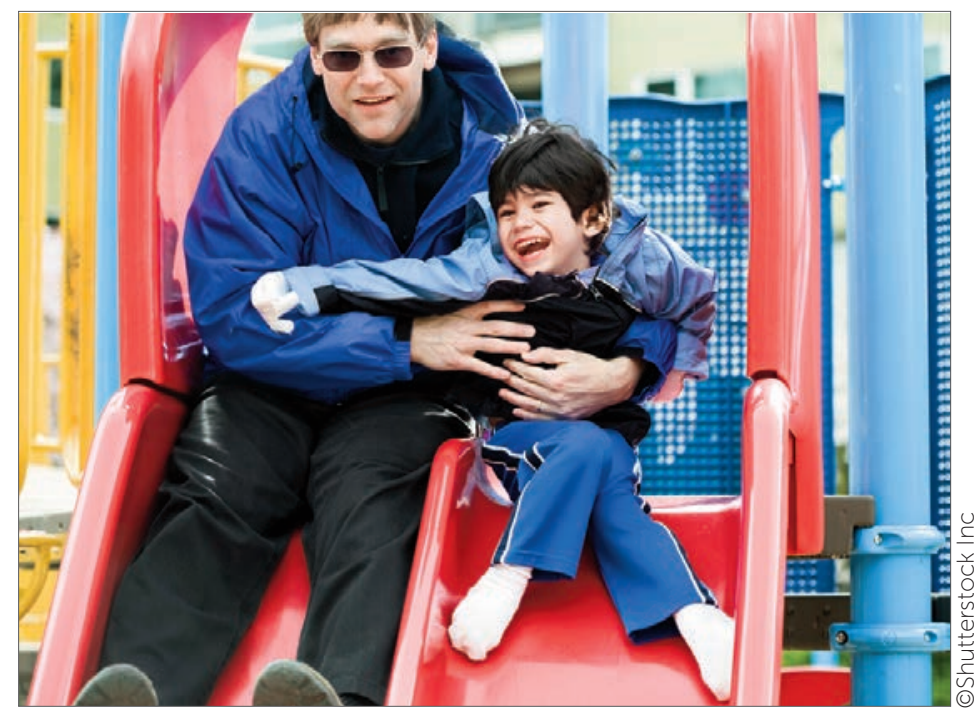

Spasticity in cerebral palsy may be treated with neuromuscular blockade

Tardieu Scale was used to estimate the contributions of spasticity and contracture $[9,10]$. For his haemophilia, Joshua received prophylaxis three-times a week with no reports of bleeds.

He presented with hypertonicity in his lower limbs, with loss of passive extensibility and dynamic overactivity in hip adductors, hamstrings and gastrocnemii. On physical examination Joshua had bilateral hot swollen anterior ankle joints as a result of the supramalleolar splints, which had been provided for ankle stability. This was worse on the left, reflecting the fact that the left ankle was tighter. This was compounded by the dynamic element of his gastrocnemii.

Functionally, Joshua was mobile indoors with the assistance of walking aids, albeit slowly and with adducted and internally rotated hips, semi-flexed knees and a toetoe gait pattern. On observation, his bare foot gait using his walking aids was slow and effortful, with a small base of support. Joshua had a bilateral equinus gait and hyperextended his knees during stance phase. There was some hip excursion, but he maintained hip flexion throughout his walking pattern and had poor pelvic stability with little coactivation of his trunk. Utilising his supramalleolar splints there was an improvement in foot clearance during swing and foot stability in stance. All other joint positions remained the same.

The GMFM-66 is a criterion-referenced observational measure designed and validated to measure change in 
TABLE 1: Joint ranges before and after (BtA)

\begin{tabular}{|c|c|c|c|c|c|c|c|}
\hline \multirow[t]{2}{*}{ Joint } & \multirow[t]{3}{*}{ Movement } & \multicolumn{6}{|c|}{ Passive range of movement (degrees) } \\
\hline & & \multicolumn{3}{|c|}{ Right } & \multicolumn{3}{|l|}{ Left } \\
\hline & & Pre & $8 \mathrm{wk}$ & 3 Months & Pre & $8 w k$ & 3 Months \\
\hline \multirow[t]{6}{*}{ Supine hip } & Flexion $(0-125)$ & Full & Full & Full & Full & Full & Full \\
\hline & Extension (Thomas test) & Negative & Negative & Negative & Negative & Negative & Negative \\
\hline & Abduction in flexion & 40 & 45 & 45 & 40 & 45 & 45 \\
\hline & Dynamic catch & 25 & No & 25 & 25 & No & 25 \\
\hline & Abduction in extension $(0-45)$ & 20 & 30 & 30 & 20 & 40 & 30 \\
\hline & Dynamic catch & 15 & no & 20 & 15 & no & 20 \\
\hline \multirow[t]{4}{*}{ Knee } & Flexion (0-140) & Full & Full & Full & Full & Full & Full \\
\hline & Extension & 0 & 0 & +5 & 0 & +5 & +5 \\
\hline & Popliteal angle unilateral & 35 & 35 & 30 & 35 & 35 & 40 \\
\hline & Dynamic catch & 60 & No & No & 60 & No & No \\
\hline \multirow[t]{3}{*}{ Ankle } & Dorsiflexion knee flexed (0-20) & 15 & 25 & 20 & 15 & 20 & 20 \\
\hline & Dorsiflexion knee extended(0-20) & 0 & 20 & 20 & 0 & 20 & 20 \\
\hline & Dynamic catch & -30 & -5 & 90 & -40 & -10 & 90 \\
\hline Spine & & & Straight & & & & \\
\hline Clonus & & & Not appa & & & & \\
\hline
\end{tabular}

gross motor function over time in children with cerebral palsy [11]. Joshua scored $83 \%$ in the crawling and kneeling dimensions and $26 \%$ in the standing dimension.

Furthermore he was classified as 'Level Il' using the Gross Motor Function Classification System (GMFCS): "Children floor sit, but may have difficulty with balance when both hands are free to manipulate objects. Movements in and out of sitting are performed without adult assistance. Children pull to stand on a stable surface. Children crawl on hands and knees with a reciprocal pattern, cruise holding on to furniture and walk using an assistive mobility device as preferred methods of mobility," [11].

\section{Treatment approach}

In consultation with the Movement Disorder Team, it was decided that intramuscular BtA was indicated to Joshua's hip adductors, bilateral hamstrings and gastrocnemii, because of dynamic contractures that were interfering with walking and wearing splints.

Collaborative goals of easing stretching, reducing scissoring and equinus during gait and increasing tolerance to orthotics were agreed. He received a review of his splints and was cast for hinged ankle foot orthoses. These were chosen as it was felt that a longer lever splint would help to improve stability at his ankle, reduce knee hyperextension and control his dynamic equinus in conjunction with the BtA. These changes would be expected to result in a more even pressure exerted by the orthotic and as such reduce his swollen ankle joints.

One day prior to Joshua's injections he commenced $250 \mathrm{mg}(15 \mathrm{mg} / \mathrm{kg})$ tranexamic acid three-times-daily and was advised to continue taking it for 7 days post procedure. Dosage of BtA injected was calculated as international consensus guidelines [3]. He received BtA (Dysport) under general anaesthetic with $1000 \mathrm{IU}$ factor VIII and 250mg tranexamic acid cover pre and post operatively for one day. The following day Joshua returned to his usual prophylaxis regime.
On discharge one day later, Joshua had no pain or bruising and the family were advised to continue with his stretching programme and local physiotherapy.

\section{Treatment outcome}

On reassessment after 8 weeks, the parents reported improvements in walking-speed, general movement and in providing for Joshua's personal care. On examination the dynamic element of his hypertonicity had either reduced or was absent, thereby assisting his fluidity and range of movement (Table 1).

On observation, his bare foot gait using his walking aids had improved in both speed and fluidity with a wider base of support. He had a bilateral toe heel gait, with improved heel contact more on the right than the left. He retained some hyperextension at his knees.

When using hinged ankle foot orthoses Joshua's walking pattern had improved, with a notable reduction in knee hyperextension and the achievement of bilateral heel strike. Functionally, the ability to stand holding on to furniture with only one hand was observed.

The GMFM-66 was not completed at this assessment, because significant functional changes would not be expected at this point.

At the 3 month follow up, Joshua's parents reported he was able to high-kneel, walk forward on his knees and had managed to stand independently for 18 seconds and on occasions had taken 5-8 steps independently. He was able to climb the stairs utilising alternate feet on each stair and his walking speed was quicker. Functionally Joshua was able to sit in the bath holding on to the rails. On the GMFM-66 he scored $95.2 \%$ on the crawling and kneeling dimension and $33 \%$ on the standing dimension: demonstrating an overall clinical improvement in function. His GMFCS remained at level II.

On observation, Joshua's bare foot gait was flat-footed with early heel rise when walking slowly and a bilateral equinus gait as he increased his speed. It was evident that 
he had retained knee hyperextension during stance with some hip flexion. When walking in orthoses he was able to achieve a bilateral heel strike, but often chose to walk with a flat footed gait, with knees in slight flexion. Pelvic stability and active hip extension during gait remained poor, but his balance and stability had improved. This was evidenced by standing and stepping and holding on to only one pod. Joshua's gait pattern remained fluid with increased speed from his initial assessment. Limited independent walking was observed. Overall, Joshua's confidence while walking bare foot or using his splints and pods had improved significantly.

\section{Conclusion}

This is the first report to our knowledge to demonstrate the benefits of using BtA in a child with severe haemophilia $A$ and a co-existing diagnosis of CP. Our clinical and formal assessments demonstrated positive changes in ambulation, tolerance of orthotics, functional abilities and musculoskeletal range of movement and no adverse bleeding side effects. The use of BtA in spasticity management is well documented in the literature for the management of hypertonicity in children with $\mathrm{CP}$ and should be considered in children with haemophilia and CP.

\section{Acknowledgements}

The authors stated that they had no interests, which might be perceived as posing a conflict or bias.

\section{References}

1. Carr LJ, Cosgrove AP, Gringas P, Neville BGR, on behalf of the UK Botulinum Toxin and cerebral palsy working party. Position paper on the use of botulinum toxin in cerebral palsy. Arch Dis Child 1998; 79: 271-273

2. Delgado MR, Hirtz D, Aisen M, et al. Practice Parameter: Pharmocologic treatment of spasticity in children and adolescents with cerebral palsy (an evidence-based review) Report of the quality standards subcommittee of the child neurology society. Neurology 2010; 74: 336-343.

3. Love SC, Novak I, Kentish M, et al. Botulinum toxin assessment, intervention and after care for lower limb spasticity in chiddren with cerebral palsy:international 4. Corry IS, Cosgrove AP, Duffy CM, et al. Botulinum toxin A compared with stretching casts in the treatment of spastic equines: a randomized prospective trial. J Ped Orthopaedics 1998, 18, 304-311.

5. Cosgrove AP, Corry IS, Graham HK. Botulinum toxin in the management of the lower limb in cerebral palsy. Dev Med Child Neurol 1994: 36: 386-396.

6. Graham HK, Aokia KR, Autti-Ramob, et al. Recommendations for the use of botulinum toxin type A in the management of cerebral palsy. Gait Posture 2000; 11 67-79

7. Ward A. Long-term modifications of spasticity. J Rehabil Med 2003; 35: 60-65 8. de Paiva A, Muenier FA, Molgo J, et al. Functional repair of motor endplates after botulinum neurotixin type A poisoning:biphasic switch of synaptic activity between nerve sprouts and their parent terminals. Proc Natl Acad Sci USA 1999; 96: 3220-5. 9. Boyd RN, Graham HK. Objective measurement of clinical findings in the use of botulinum toxin type A for the management of children with cerebral palsy. European JNeurol 1999; 6 (Suppl): 23-35.

10. Ashworth B. Preliminary trial of carisoprodal in multiple sclerosis. Practitioner 1964; 192: 540-542.

11. Palisanao R, Rosenbaum $P$, Walter $S$, et al Development and reliability of a system to classify gross motor function in children with cerebral palsy. Dev Med Child Neurol 1997; 39: 214-233.

\section{The Journal of} Haemophilia Practice

\section{An open-access} journal for sharing

experience in

the care of

people with

bleeding

disorders

www.haemjournal.com 